$$
\begin{array}{lllll}
\lrcorner & \lrcorner
\end{array}
$$

On a conjecture of Kontsevich and Soibelman

\lrcorner

\rfloor

.

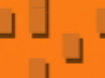

$$
\lrcorner\lrcorner \_ \text {Lê Quy Thuong }
$$




\title{
On a conjecture of Kontsevich and Soibelman
}

\author{
Lê Quy Thuong
}

Dedicated to Professor Hà Huy Vui on the occasion of his sixtieth birthday

\begin{abstract}
We consider a conjecture of Kontsevich and Soibelman which is regarded as a foundation of their theory of motivic Donaldson-Thomas invariants for noncommutative $3 d$ Calabi-Yau varieties. We will show that, in some certain cases, the answer to this conjecture is positive.
\end{abstract}

\section{Introduction}

Kontsevich and Soibelman [2008] introduce and give discussions on the motivic Donaldson-Thomas invariants which are defined for noncommutative $3 d$ CalabiYau varieties and take values in certain Grothendieck groups of algebraic varieties. One of the main objectives of Kontsevich and Soibelman's paper is to define the motivic Hall algebra which generates Toën's notion [2006] of the derived Hall algebra. For $\mathscr{C}$ an ind-constructible triangulated $A_{\infty}$-category over a field $\kappa$, the motivic Hall algebra $H(\mathscr{C})$ is constructed to become a graded associative algebra, which admits for each strict sector $V$ an element $A_{V}^{\mathrm{Hall}}$ invertible in the completed motivic Hall algebra and satisfying the factorization property. It is believed that, in the case of $3 d$ Calabi-Yau category, there is a homomorphism $\Phi$ of the motivic Hall algebra into the motivic quantum torus defined in terms of the motivic Milnor fiber of the potential. Then the motivic Donaldson-Thomas invariants appear as the collection of the images of $A_{V}^{\text {Hall }}$ under the homomorphism $\Phi$.

In fact, the following conjecture plays a central role in the existence of $\Phi$. Assume that the characteristic of $\kappa$ is zero. Let $F$ be a formal series on the affine space $\mathbb{A}_{\kappa}^{d}=\mathbb{A}_{\kappa}^{d_{1}} \times{ }_{\kappa} \mathbb{A}_{\kappa}^{d_{2}} \times{ }_{\kappa} \mathbb{A}_{\kappa}^{d_{3}}$, depending in a constructible way on finitely many extra parameters, such that $F(0,0,0)=0$ and $F$ has degree zero with respect to the diagonal action of the multiplicative group $\mathbb{G}_{m, \kappa}$ with the weights $(1,-1,0)$. In particular, $F(x, 0,0)$ is the zero function on $\mathbb{A}_{\kappa}^{d_{1}}$. We denote by $X_{0}(F)$ the set of the zeros of $F$ on $\mathbb{A}_{\kappa}^{d}$. Consider the natural inclusions

MSC2010: primary 14B05; secondary 14B07, 14J17, 32S05, 32S30, $32 \mathrm{~S} 55$.

Keywords: arc spaces, motivic Milnor fiber, motivic zeta function, Newton polyhedron. 


$$
i_{1}: \mathbb{A}_{\kappa}^{d_{1}} \times_{\kappa} \mathbb{G}_{m, \kappa} \rightarrow X_{0}(F) \times_{\kappa} \mathbb{G}_{m, \kappa} \quad \text { and } \quad i_{0}:\{0\} \times_{\kappa} \mathbb{G}_{m, \kappa} \rightarrow X_{0}(F) \times_{\kappa} \mathbb{G}_{m, \kappa} .
$$

Consider the motivic Milnor fiber $\mathscr{S}_{F}$ of $F$ in the ring $\mathcal{M}_{X_{0}(F) \times_{\kappa} \mathbb{G}_{m, \kappa}}^{\mathbb{G}_{m, \kappa}}$, the localization of the relative Grothendieck ring defined in [Guibert et al. 2005; 2006]. Denote by $h$ the function on $\mathbb{A}_{\kappa}^{d_{3}}$ defined by $h(z)=F(0,0, z)$. We write $\mathscr{S}_{h, 0}$ for the pullback $i_{0}^{*} \mathscr{\varphi}_{h}$. We denote by the integral $\int_{\mathbb{A}_{K}}$ the pushforward of the canonical morphism $\pi: \mathbb{A}_{\kappa}^{d_{1}} \times{ }_{\kappa} \mathbb{G}_{m, \kappa} \rightarrow \operatorname{Spec}(k) \times{ }_{\kappa} \mathbb{G}_{m, \kappa}$.

Conjecture 1.1 [Kontsevich and Soibelman 2008]. With the previous notations and hypotheses, the following formula holds in $M_{\mathbb{G}_{m, \kappa}}^{\mathbb{G}_{m, \kappa}}$ :

$$
\int_{\mathbb{A}_{\kappa}^{d_{1}}} i_{1}^{*} \mathscr{\varphi}_{F}=\mathbb{L}^{d_{1}} \mathscr{Y}_{h, 0} .
$$

In this paper, we consider the conjecture in some special (actually quite general) cases, namely, when $F$ is a composition of a polynomial in two variables and a pair of two regular functions (Theorem 5.1), or $F$ has the form

$$
F(x, y, z)=g(x, y, z)+h(z)^{\ell}
$$

with $\ell$ sufficiently large (function of Steenbrink type, Theorem 5.6) under some additional conditions of nondegeneracy with respect to its Newton polyhedron (this would be the general case for the conjecture if we did not assume $\ell$ sufficiently large). For these cases, we use previous results of Guibert, Loeser and Merle [Guibert et al. 2006; 2009] for the motivic Milnor fiber of composite functions or functions of Steenbrink type. We also use in an important way, via Proposition 4.8, the explicit computation of the motivic Milnor fiber of a regular function via its Newton polyhedron (suggested by [Guibert 2002]). These lead to the positive answer to the conjecture in the cases considered.

\section{Motivic zeta function and motivic Milnor fiber}

Let us recall some basic notations in the theory of motivic integration which will be used in this paper. For references, we follow [Denef and Loeser 1998, 1999a; 2001; Guibert 2002; Guibert et al. 2005; 2006].

2A. Let $\kappa$ be a field of characteristic zero. For a variety $X$ over $\kappa$, we denote by $\mathscr{L}_{m}(X)$ the space of $m$-arcs on $X$, and by $\mathscr{L}(X)$ a limit of the projective system of spaces $\mathscr{L}_{m}(X)$ and (canonical) morphisms $\mathscr{L}_{l}(X) \rightarrow \mathscr{L}_{m}(X)(l \geq m)$. In this paper, we use the notation $\pi_{m}$ for the canonical morphism $\mathscr{L}(X) \rightarrow \mathscr{L}_{m}(X)$. The $\mathbb{G}_{m, \kappa}$-action on $\mathscr{L}_{m}(X)$ and $\mathscr{L}(X)$ is given by $a \cdot \varphi(t)=\varphi(a t)$. The notation $\mathcal{M}_{X}$ can be found in [Guibert et al. 2006]. As in [Guibert et al. 2005], we denote by $M_{X \times_{\kappa} \mathbb{G}_{m, \kappa}}^{\mathbb{G}_{m, k}}$ the localization at $\mathbb{L}$ of the relative Grothendieck ring of $\mathbb{G}_{m, \kappa}$-equivariant 
morphisms $Y \rightarrow X \times{ }_{\kappa} \mathbb{G}_{m, \kappa}$ endowed with a monomial $\mathbb{G}_{m, \kappa}$-action, where $\mathbb{L}$ is the class of the line bundle $\mathbb{A}_{X \times_{\kappa} \mathbb{G}_{m, \kappa}}^{1}$.

From now on, the group scheme $\mathbb{G}_{m, \kappa}=\operatorname{Spec}\left(\kappa\left[t, t^{-1}\right]\right)$ will be written simply as $\mathbb{G}$.

2B. Motivic zeta function and motivic Milnor fiber. Let $X$ be a smooth variety over $\kappa$ of pure dimension $n$, and let $g: X \rightarrow \mathbb{A}_{\kappa}^{1}$ be a function on $X$, with zero locus $X_{0}(g)$. For $m \geq 1$, we define

$$
\mathscr{L}_{m}(g):=\left\{\varphi \in \mathscr{L}_{m}(X) \mid \operatorname{ord}_{t} g(\varphi)=m\right\} .
$$

Note that this variety is invariant by the $\mathbb{G}$-action on $\mathscr{L}_{m}(X)$. Furthermore, $g$ induces a morphism $g_{m}: \mathscr{L}_{m}(g) \rightarrow \mathbb{G}$, assigning to a point $\varphi$ in $\mathscr{L}_{m}(X)$ the coefficient $\operatorname{ac}(g(\varphi))$ of $t^{m}$ in $g(\varphi(t))$, which we also denote by $\operatorname{ac}(g)(\varphi)$. This morphism is a diagonally monomial of weight $m$ with respect to the $\mathbb{G}$-action on $\mathscr{X}_{m}(g)$ since $g(s \cdot \varphi)=s^{m} g_{m}(\varphi)$. We thus consider the class $\left[\mathscr{X}_{m}(g)\right]$ of $\mathscr{X}_{m}(g)$ in $\mathcal{M}_{X_{0}(g) \times_{k} \mathbb{G}}^{\mathbb{G}}$. We can now consider the motivic zeta function

$$
Z_{g}(T):=\sum_{m \geq 1}\left[\mathscr{Q}_{m}(g)\right] \mathbb{L}^{-m n} T^{m}
$$

in $M_{X_{0}(g) \times_{k} \mathbb{G}}^{\mathbb{G}} \llbracket T \rrbracket$. Note that $Z_{g}=0$ if $g=0$ on $X$.

By using a log-resolution of $X_{0}(g)$, Denef and Loeser [1998; 2001] proved that $Z_{g}(T)$ is a rational series in $M_{X_{0}(g) \times_{k} \mathbb{G}}^{\mathbb{G}} \llbracket T \rrbracket_{s r}$ (see next paragraph) and they also showed that one can consider the $\operatorname{limit}_{T \rightarrow \infty} Z_{g}(T)$ in $M_{X_{0}(g) \times_{k} \mathbb{G}}^{\mathbb{G}}$. Then the motivic Milnor fiber of $g$ is defined as

$$
\mathscr{Y}_{g}:=-\lim _{T \rightarrow \infty} Z_{g}(T) .
$$

2C. Rational series and their limits. Let $A$ be one of the rings

$$
\mathbb{Z}\left[\mathbb{L}, \mathbb{L}^{-1}\right], \quad \mathbb{Z}\left[\mathbb{L}, \mathbb{L}^{-1},\left(1 /\left(1-\mathbb{L}^{-i}\right)\right)_{i>0}\right], \quad \mu_{S \times_{k} \mathbb{G}}^{\mathbb{G}} .
$$

We denote by $A \llbracket T \rrbracket_{s r}$ the $A$-submodule of $A \llbracket T \rrbracket$ generated by 1 and by finite products of terms $p_{e, i}(T)=\mathbb{L}^{e} T^{i} /\left(1-\mathbb{L}^{e} T^{i}\right)$ with $e$ in $\mathbb{Z}$ and $i$ in $\mathbb{N}_{>0}$. There is a unique $A$-linear morphism

$$
\lim _{T \rightarrow \infty}: A \llbracket T \rrbracket_{s r} \rightarrow A
$$

such that

$$
\lim _{T \rightarrow \infty}\left(\prod_{i \in I} p_{e_{i}, j_{i}}(T)\right)=(-1)^{|I|}
$$

for every family $\left(\left(e_{i}, j_{i}\right)\right)_{i \in I}$ in $\mathbb{Z} \times \mathbb{N}_{>0}$ with $I$ finite (possibly empty). 
We will use the notation

$$
\begin{aligned}
& \mathbb{R}_{\geq 0}^{I}:=\left\{a=\left(a_{1}, \ldots, a_{n}\right) \in \mathbb{R}_{\geq 0}^{n} \mid a_{i}=0 \text { for } i \notin I\right\}, \\
& \mathbb{R}_{>0}^{I}:=\left\{a=\left(a_{1}, \ldots, a_{n}\right) \in \mathbb{R}_{\geq 0}^{n} \mid a_{i}=0 \Longleftrightarrow i \notin I\right\},
\end{aligned}
$$

for $I$ a subset of $\{1, \ldots, n\}$. The sets $\mathbb{Z}_{\geq 0}^{I}, \mathbb{Z}_{>0}^{I}$ and $\mathbb{N}_{>0}^{I}$ are defined similarly.

Let $\Delta$ be a rational polyhedral convex cone in $\mathbb{R}_{>0}^{I}$ and let $\bar{\Delta}$ denote its closure in $\mathbb{R}_{\geq 0}^{I}$ with $I$ a finite set. Let $l$ and $l^{\prime}$ be two integer linear forms on $\mathbb{Z}^{I}$ positive on $\bar{\Delta} \backslash\{0\}$. Consider the series

$$
S_{\Delta, l, l^{\prime}}(T):=\sum_{k \in \Delta \cap \mathbb{N}_{>0}^{I}} \mathbb{L}^{-l^{\prime}(k)} T^{l(k)}
$$

in $\mathbb{Z}\left[\mathbb{L}, \mathbb{L}^{-1}\right] \llbracket T \rrbracket$.

Lemma 2.1 [Guibert 2002]. With previous notations and hypotheses, assuming that $\Delta$ is open in its linear span $\bar{\Delta}$, the series $S_{\Delta, l, l^{\prime}}(T)$ lies in $\mathbb{Z}\left[\mathbb{L}, \mathbb{L}^{-1}\right] \llbracket T \rrbracket_{s r}$ and

$$
\lim _{T \rightarrow \infty} S_{\Delta, l, l^{\prime}}(T)=(-1)^{\operatorname{dim}(\Delta)} \text {. }
$$

\section{The Newton polyhedron of a regular function}

3A. Newton polyhedra. Let $g(x)=\sum_{\alpha \in \mathbb{N}^{n}} a_{\alpha} x^{\alpha}$ be a polynomial in $n$ variables $x=\left(x_{1}, \ldots, x_{n}\right)$ such that $g(0)=0$. We denote by $\operatorname{supp}(g)$ the set of exponents $\alpha$ in $\mathbb{N}^{n}$ with $a_{\alpha} \neq 0$. The Newton polyhedron $\Gamma$ of $g$ is the convex hull of $\operatorname{supp}(g)+\mathbb{R}_{\geq 0}^{n}$. For a compact face $\gamma$ of $\Gamma$, we denote by $g_{\gamma}$ the quasihomogenous polynomial

$$
g_{\gamma}(x)=\sum_{\alpha \in \gamma} a_{\alpha} x^{\alpha}
$$

We say $g$ is nondegenerate with respect to its Newton polyhedron $\Gamma$ if, for every compact face $\gamma$ of $\Gamma$, the face function $g_{\gamma}$ is smooth on $\mathbb{G}^{n}$.

To the Newton polyhedron $\Gamma$ we associate a function $l_{\Gamma}$ which assigns to a vector $a$ in $\mathbb{R}_{\geq 0}^{n}$ the value $\inf _{b \in \Gamma}\langle a, b\rangle$, with $\langle a, b\rangle$ being the standard inner product of $a$ and $b$. For $a$ in $\mathbb{R}_{\geq 0}^{n}$, we denote by $\gamma_{a}$ the face of $\Gamma$ on which the restriction of the function $\langle a,$.$\rangle on \Gamma$ attains its minimum, i.e., $b \in \Gamma$ is in $\gamma_{a}$ if and only if

$$
\langle a, b\rangle=l_{\Gamma}(a)=\min _{b \in \Gamma}\langle a, b\rangle .
$$

For $a=0$ in $\mathbb{R}_{\geq 0}^{n}, \gamma_{a}=\Gamma$. If $a \neq 0, \gamma_{a}$ is a proper face of $\Gamma$. Furthermore, $\gamma_{a}$ is a compact face of $\Gamma$ if and only if $a$ is in $\mathbb{R}_{>0}^{n}$. For any face $\gamma$ of the Newton polyhedron $\Gamma$, we denote by $\sigma(\gamma)$ the cone $\left\{a \in \mathbb{R}_{\geq 0}^{n} \mid \gamma_{a}=\gamma\right\}$. Its closure is given by $\bar{\sigma}(\gamma)=\left\{a \in \mathbb{R}_{\geq 0}^{n} \mid \gamma_{a} \supset \gamma\right\}$.

A fan $\mathscr{F}$ is a finite set of rational polyhedral cones such that every face of a cone of $\mathscr{F}$ is also a cone of $\mathscr{F}$, and the intersection of two arbitrary cones of $\mathscr{F}_{F}$ is the common face of them. 
3B. Partition of $\mathbb{R}_{\geq 0}^{n_{1}} \times \mathbb{R}_{>0}^{n_{2}}$ with respect to g. Write $n=n_{1}+n_{2}$ with $n_{1} \geq 0$, $n_{2} \geq 0$. Let $g$ be a function on $\mathbb{A}_{\kappa}^{n}$ that is nondegenerate with respect to the Newton polyhedron $\Gamma$ of $g$. Let $\gamma$ be a compact face of $\Gamma$. A proper face $\epsilon$ of $\Gamma$ is said to lean on $\gamma$ if there exists a subset $I$ of $\{1, \ldots, n\}$ such that

$$
\epsilon=\gamma+\mathbb{R}_{\geq 0}^{I}=\left\{a+b \mid a \in \gamma, b \in \mathbb{R}_{\geq 0}^{I}\right\} .
$$

Note that $\operatorname{dim}(\epsilon)=\operatorname{dim}(\gamma)+|I|$. Clearly, the face $\epsilon$ is noncompact when $I$ is nonempty. The following lemmas are trivial.

Lemma 3.1. If $\gamma+\mathbb{R}_{\geq 0}^{I}$ is a face leaning on a compact face $\gamma$ of $\Gamma$, then for every $J$ subset of $I, \gamma+\mathbb{R}_{\geq 0}^{J}$ is also a face of $\Gamma$ leaning on $\gamma$.

Notice that if $I=\varnothing$, the face $\gamma+\mathbb{R}_{\geq 0}^{I}$ reduces to the compact face $\gamma$. If $\epsilon=\gamma+\mathbb{R}_{\geq 0}^{I}$, we denote $\sigma_{\gamma, I}:=\sigma(\epsilon)$. It is clear that $\operatorname{dim}\left(\sigma_{\gamma, I}\right)=n-|I|-\operatorname{dim}(\gamma)$.

Lemma 3.2. If $\sigma_{\gamma, I}$ is contained in $\mathbb{R}_{\geq 0}^{n_{1}} \times \mathbb{R}_{>0}^{n_{2}}$, then for every subset $J$ of $I, \sigma_{\gamma, J}$ is contained in $\mathbb{R}_{\geq 0}^{n_{1}} \times \mathbb{R}_{>0}^{n_{2}}$. Moreover, $\sigma_{\gamma, I}$ is a face of $\sigma_{\gamma, J}$.

Lemma 3.3. Assume that $\gamma$ is a compact face and $\epsilon=\gamma+\mathbb{R}^{I}$ is a face of $\Gamma$. Then $\sigma_{\gamma, I}$ is contained in $\mathbb{R}_{\geq 0}^{n_{1}} \times \mathbb{R}_{>0}^{n_{2}}$ if and only if $I$ is a subset of $\left\{1, \ldots, n_{1}\right\}$.

Fix a compact face $\gamma$ of $\Gamma$. Let $M$ be a maximal element (in the inclusion relation) of the family of the subsets of $\left\{1, \ldots, n_{1}\right\}$ such that $\gamma+\mathbb{R}_{\geq 0}^{M}$ is a face of $\Gamma$ (thus, by Lemma 3.3, $\sigma_{\gamma, M}$ is contained in $\mathbb{R}_{\geq 0}^{n_{1}} \times \mathbb{R}_{>0}^{n_{2}}$ ). Then, for every subset $I$ of $M$, $\gamma+\mathbb{R}_{\geq 0}^{I}$ is a face of $\Gamma$ due to Lemma 3.1 , and $\sigma_{\gamma, I}$ is contained in $\mathbb{R}_{\geq 0}^{n_{1}} \times \mathbb{R}_{>0}^{n_{2}}$ by Lemma 3.2. We thus have proved the following result.

Proposition 3.4. There exists a canonical fan in $\mathbb{R}_{\geq 0}^{n_{1}} \times \mathbb{R}_{>0}^{n_{2}}$ with respect to $g$ partitioning it into the cones $\sigma_{\gamma, I}$, where I runs over the subsets of $M, M$ runs over the maximal subsets of $\left\{1, \ldots, n_{1}\right\}$ such that $\gamma+\mathbb{R}_{\geq 0}^{M}$ is a face of $\Gamma$, and $\gamma$ runs over the compact faces of $\Gamma$.

Example 3.5. Consider a function $g\left(x_{1}, \ldots, x_{n}\right)$ with $\Gamma_{g}$ having a unique vertex $P$. Then the $k$-dimensional faces of $\Gamma$ leaning on $P$ have the form

$$
P+\mathbb{R}_{\geq 0}^{I}
$$

with $I$ a subset of $\{1, \ldots, n\}$ and $|I|=k$, for $k=0, \ldots, n-1$. We deduce from Lemma 3.3 that the canonical partition of $\mathbb{R}_{\geq 0}^{n_{1}} \times \mathbb{R}_{>0}^{n_{2}}$ with respect to $g$ is given by the cones $\sigma_{P, I}$, with $I$ a subset of $\left\{1, \ldots, n_{1}\right\}$.

Remark 3.6. In the case $n_{1}=0$, we reduce to the work in [Guibert 2002]. More clearly, for each compact face $\gamma$ of $\Gamma$, all the maximal subsets $M$ of $\{1, \ldots, n\}$, of which $\gamma+\mathbb{R}_{\geq 0}^{M}$ is a face of $\Gamma$ and $\sigma_{\gamma, M} \subset \mathbb{R}_{>0}^{n}$, are empty. 


\section{Computation of $i_{1}^{*} \mathscr{Y}_{g}$ and $\int_{\mathbb{A}_{\kappa}} i_{1}^{*} \mathscr{Y}_{g}$}

Consider a regular function $g$ on $\mathbb{A}_{\kappa}^{n}$. We assume that $g$ is nondegenerate with respect to its Newton polyhedron $\Gamma$. Denote by $i_{1}$ the natural inclusion $\mathbb{A}_{\kappa}^{n_{1}} \hookrightarrow \mathbb{A}_{\kappa}^{n}$ or $\mathbb{A}_{\kappa}^{n_{1}} \times_{\kappa} \mathbb{G} \hookrightarrow \mathbb{A}_{\kappa}^{n} \times{ }_{\kappa} \mathbb{G}$.

4A. The motivic zeta function $Z_{g}(T)$. We identify the arc space $\mathscr{L}\left(\mathbb{A}_{\kappa}^{n}\right)$ with the space of formal power series $\kappa \llbracket t \rrbracket^{n}$ via the system of coordinates $x_{1}, \ldots, x_{n}$. For every $\operatorname{arc} \varphi \in \mathscr{L}\left(\mathbb{A}_{\kappa}^{n}\right)$, we note $\operatorname{ord}_{t} x(\varphi)=\left(\operatorname{ord}_{t} x_{1}(\varphi), \ldots, \operatorname{ord}_{t} x_{n}(\varphi)\right)$. For every $m \in \mathbb{N}_{>0}$ and $a \in \mathbb{N}^{n}$ we set

$$
\mathscr{X}_{a, m}(g)=\mathscr{X}_{m}(g) \cap \pi_{m}\left(\mathscr{X}_{a}\right),
$$

where the spaces $\mathscr{X}_{m}(g)$ and $\mathscr{X}_{a}$ are defined as follows:

$$
\begin{aligned}
\mathscr{X}_{m}(g) & =\left\{\varphi \in \mathscr{L}_{m}\left(\mathbb{A}_{\kappa}^{n}\right) \mid \operatorname{ord}_{t} g(\varphi)=m\right\}, \\
\mathscr{X}_{a} & =\left\{\varphi \in \mathscr{L}\left(\mathbb{A}_{\kappa}^{n}\right) \mid \operatorname{ord}_{t} x(\varphi)=a\right\} .
\end{aligned}
$$

It is clear that $\mathscr{L}_{a, m}(g)$ is a variety over $X_{0}(g) \times_{\kappa} \mathbb{G}$ in which the morphism to $X_{0}(g)$ is induced by the canonical morphism $\mathscr{L}_{m}\left(\mathbb{A}_{\kappa}^{n}\right) \rightarrow \mathbb{A}_{\kappa}^{n}$ and the morphism to $\mathbb{G}$ is the morphism ac $(g)$. Note that $\mathscr{L}_{a, m}(g)$ is invariant by the $\mathbb{G}$-action on $\mathscr{L}_{m}\left(\mathbb{A}_{\kappa}^{n}\right)$.

For every $a \in \mathbb{N}^{n}$ and $\varphi \in \mathscr{X}_{a}, \operatorname{ord}_{t} g(\varphi) \geq l_{\Gamma}(a)$ by the definition of $l_{\Gamma}$. Furthermore, $\mathscr{X}_{m}(g)$ can be expressed as a disjoint union $\bigcup_{a \in \mathbb{N}^{n}} \mathscr{X}_{a, m}(g)$ of the subspaces $\mathscr{X}_{a, m}(g)$ for $a$ in $\mathbb{N}^{n}$. Then the motivic zeta function $Z_{g}(T)$ of $g$ can be written in the following form:

$$
\begin{aligned}
Z_{g}(T) & =\sum_{a \in \mathbb{N}^{n}} \sum_{m \geq l_{\Gamma}(a)}\left[\mathscr{X}_{a, m}(g)\right] \mathbb{L}^{-n m} T^{m} \\
& =\sum_{a \in \mathbb{N}^{n}}\left(\left[\mathscr{X}_{a, l_{\Gamma}(a)}(g)\right] \mathbb{L}^{-n l_{\Gamma}(a)} T^{l_{\Gamma}(a)}+\sum_{m \geq l_{\Gamma}(a)+1}\left[\mathscr{X}_{a, m}(g)\right] \mathbb{L}^{-n m} T^{m}\right) \\
& =: Z^{0}(T)+Z^{1}(T) .
\end{aligned}
$$

There is a canonical partition of $\mathbb{R}_{\geq 0}^{n}$ into the rational polyhedral cones $\sigma(\gamma)$ with $\gamma$ running over the proper faces of $\Gamma$, so we deduce that

$$
\begin{aligned}
& Z^{0}(T)=\sum_{\gamma} \sum_{a \in \sigma(\gamma)}\left[\mathscr{X}_{a, l_{\Gamma}(a)}(g)\right] \mathbb{L}^{-n l_{\Gamma}(a)} T^{l_{\Gamma}(a)}, \\
& Z^{1}(T)=\sum_{\gamma} \sum_{a \in \sigma(\gamma)} \sum_{k \geq 1}\left[\mathscr{X}_{a, l_{\Gamma}(a)+k}(g)\right] \mathbb{L}^{-n\left(l_{\Gamma}(a)+k\right)} T^{l_{\Gamma}(a)+k},
\end{aligned}
$$

where the sum $\sum_{\gamma}$ runs over the proper faces $\gamma$ of $\Gamma$. 
4B. Computation of $i_{1}^{*} Z_{g}(T)$. Assume that $g$ satisfies the additional condition that $\mathbb{A}_{\kappa}^{n_{1}}$ is naturally included in $X_{0}(g)$ via the morphism $i_{1}$. To compute $i_{1}^{*} Z_{g}(T)$, we consider the canonical fan in $\mathbb{R}_{>0}^{n_{1}} \times \mathbb{R}_{>0}^{n_{2}}$ with respect to $g$. Denote by $\Gamma_{c}$ the set of compact faces of $\Gamma$ and by $\mathfrak{M}_{\gamma}$ the set of maximal subsets $M$ of $\left\{1, \ldots, n_{1}\right\}$ such that $\gamma+\mathbb{R}_{\geq 0}^{M}$ is a face of $\Gamma$. By Proposition 3.4, we can partition $\mathbb{R}_{\geq 0}^{n_{1}} \times \mathbb{R}_{>0}^{n_{2}}$ into the cones $\sigma_{\gamma, I}$, with $I$ a subset of $M, M$ in $\mathfrak{M}_{\gamma}$ and $\gamma$ in $\Gamma_{c}$. Assume that $\mathfrak{M}_{\gamma}=\left\{M_{1}, \ldots, M_{p}\right\}$. We denote by $\mathfrak{S}_{\gamma}$ the family of subsets of one of the sets $M_{1}, \ldots, M_{p}$. Then we have

$$
\begin{aligned}
& i_{1}^{*} Z^{0}(T)=i_{1}^{*}\left(\sum_{\gamma \in \Gamma_{c}} \sum_{I \in \mathfrak{S}_{\gamma}} \sum_{a \in \sigma_{\gamma, I}}\left[\mathscr{Q}_{a, l_{\Gamma}(a)}(g)\right] \mathbb{L}^{-n l_{\Gamma}(a)} T^{l_{\Gamma}(a)}\right), \\
& i_{1}^{*} Z^{1}(T)=i_{1}^{*}\left(\sum_{\gamma \in \Gamma_{c}} \sum_{I \in \mathfrak{S}_{\gamma}} \sum_{a \in \sigma_{\gamma, I}} \sum_{k \geq 1}\left[\mathscr{X}_{a, l_{\Gamma}(a)+k}(g)\right] \mathbb{L}^{-n\left(l_{\Gamma}(a)+k\right)} T^{l_{\Gamma}(a)+k}\right) .
\end{aligned}
$$

4C. Class of $\mathscr{X}_{\boldsymbol{a}, \boldsymbol{m}}(\boldsymbol{g})$. For a compact face $\gamma$ of $\Gamma$, consider the variety $X_{\gamma}:=$ $\mathbb{G}^{n} \backslash g_{\gamma}^{-1}(0)$ endowed with a $\mathbb{G}$-action as follows: if $\gamma=\gamma_{a}, a=\left(a_{1}, \ldots, a_{n}\right)$ then we set

$$
s \cdot\left(\xi_{1}, \ldots, \xi_{n}\right)=\left(s^{a_{1}} \xi_{1}, \ldots, s^{a_{n}} \xi_{n}\right) .
$$

For each compact $\gamma$ and $I$ in $\mathfrak{S}_{\gamma}$, consider the morphism

$$
g_{\gamma, I}: X_{\gamma}=\mathbb{G}^{n} \backslash g_{\gamma}^{-1}(0) \rightarrow X_{0}(g) \times_{\kappa} \mathbb{G}
$$

given by

$$
g_{\gamma, I}\left(\xi_{1}, \ldots, \xi_{n}\right)=\left(\left(\hat{\xi}_{1}, \ldots, \hat{\xi}_{n}\right), g_{\gamma}\left(\xi_{1}, \ldots, \xi_{n}\right)\right),
$$

where $\hat{\xi}_{i}$ is defined by

$$
\hat{\xi}_{i}= \begin{cases}\xi_{i} & \text { if } i \in I \\ 0 & \text { otherwise. }\end{cases}
$$

The first projection $X_{\gamma} \rightarrow X_{0}(g)$ is $\mathbb{G}$-equivariant in an obvious manner, and for $\gamma=\gamma_{a}$, the second $X_{\gamma} \rightarrow \mathbb{G}$ is diagonally monomial of weight $l_{\Gamma}(a)$ with respect to the $\mathbb{G}$-action since $g_{\gamma}\left(s \cdot\left(\xi_{1}, \ldots, \xi_{n}\right)\right)=s^{l_{\Gamma}(a)} g_{\gamma}\left(\xi_{1}, \ldots, \xi_{n}\right)$ for any $s$ in $\mathbb{G}$. This defines a class $\left[g_{\gamma, I}: X_{\gamma} \rightarrow X_{0}(g) \times_{\kappa} \mathbb{G}\right]$ in $M_{X_{0}(g) \times_{\kappa} \mathbb{G}}^{\mathbb{G}}$, which we denote by $\Phi_{\gamma, I}$. Notice that $\Phi_{\gamma, I}$ does not depend on the action thanks to the construction of the Grothendieck group (cf. [Guibert et al. 2005, 2006]).

We denote by $\Psi_{\gamma, I}$ the class in $M_{X_{0}(g) \times_{\kappa} \mathbb{G}}^{\mathbb{G}}$ of the morphism

$$
g_{\gamma}^{-1}(0) \times{ }_{\kappa} \mathbb{G} \rightarrow X_{0}(g) \times{ }_{\kappa} \mathbb{G},
$$

which maps $\left(\left(\xi_{1}, \ldots, \xi_{n}\right), t\right)$ to $\left(\left(\hat{\xi}_{1}, \ldots, \hat{\xi}_{n}\right), t^{l_{\Gamma}(a)}\right)$ for $\gamma=\gamma_{a}$, with the $\mathbb{G}$-action on $g_{\gamma, I}^{-1}(0)$ given by $s \cdot\left(\xi_{1}, \ldots, \xi_{n}\right)=\left(s^{a_{1}} \xi_{1}, \ldots, s^{a_{n}} \xi_{n}\right)$, the $\mathbb{G}$-action on $\mathbb{G}$ given by the multiplicative translation, $g_{\gamma}^{-1}(0) \times_{\kappa} \mathbb{G} \rightarrow X_{0}(g)$ being $\mathbb{G}$-equivariant, and 
$g_{\gamma}^{-1}(0) \times{ }_{\kappa} \mathbb{G} \rightarrow \mathbb{G}$ being diagonally monomial of weight $l_{\Gamma}(a)$ with respect to the $\mathbb{G}$-action.

Lemma 4.1. The following formulas hold in $M_{X_{0}(g) \times_{k} \mathbb{G}}^{\mathbb{G}}$ for every a in $\sigma_{\gamma, I}$ :

(i) If there is a nonempty subset $I$ of $\{1, \ldots, n\}$ such that $a_{i}>m$ for any $i \in I$ and $\left.g\right|_{\mathbb{A}_{\kappa}^{c}}=0$, then $\left[\mathscr{X}_{a, m}(g)\right]=0$.

If $a_{i} \leq l_{\Gamma}(a)$ for any $i=1, \ldots, n$, we have

(ii) $\left[\mathscr{Q}_{a, l_{\Gamma}(a)}(g)\right]=\Phi_{\gamma, I} \mathbb{L}^{n l_{\Gamma}(a)-s(a)}$,

(iii) $\left[\mathscr{X}_{a, l_{\Gamma}(a)+k}(g)\right]=\Psi_{\gamma, I} \mathbb{L}^{n\left(l_{\Gamma}(a)+k\right)-s(a)}$ for $k \geq 1$.

Here, $\mathbb{A}_{\kappa}^{I^{c}}:=\left\{\left(x_{1}, \ldots, x_{n}\right) \in \mathbb{A}_{\kappa}^{n} \mid x_{i}=0 \forall i \in I\right\}$, and $s(a):=\sum_{i=1}^{n} a_{i}$.

Proof. Item (i) follows from the definition of $\mathscr{X}_{a, m}(g)$ and from the hypothesis on $g$. Indeed, every element of $\pi_{m}\left(\mathscr{C}_{a}\right)$ has the form $\varphi(t)=\left(x_{1}(t), \ldots, x_{n}(t)\right)$, where $x_{j}(t)$ is a polynomial of degree $\leq m$ in a variable $t$ for any $j=1, \ldots, n$, and $x_{i}(t)$ is the zero polynomial if $i$ is in $I$. Then $g(\varphi(t))=0$ and $\operatorname{ord}_{t} g(\varphi)=\infty$, which means that $\mathscr{X}_{a, m}(g)=\varnothing$.

Items (ii) and (iii) may be deduced from the proofs of [Guibert 2002, Lemmas 2.1.1, 2.1.2] and from the isomorphism $\mu_{X_{0}(g)}^{\hat{\mu}} \cong M_{X_{0}(g) \times \mathbb{G}}^{\mathbb{G}}$ (cf. [Guibert et al. 2006, Proposition 2.6]). In [Guibert 2002, Section 2.1] (in particular, Lemmas 2.1.1 and 2.1.2), Guibert only considers functions of the form $\sum_{\alpha \in \mathbb{N}_{>0}^{n}} f_{\alpha} x^{\alpha}$. Observe that his condition that $\alpha \in \mathbb{N}_{>0}^{n}$ is equivalent to the condition that $a_{i} \leq l_{\Gamma}(a)$ for any $i=1, \ldots, n$. Finally, notice that the hypothesis of nondegeneracy with respect to $\Gamma$ is in fact the main tool for the proofs.

There is also a way to prove (ii) directly as follows. An element $\varphi(t)$ of $\mathscr{X}_{a, l_{\Gamma}(a)}(g)$ has the form $\varphi(t)=\left(x_{1}(t), \ldots, x_{n}(t)\right)$, where $x_{i}(t)=\sum_{m=a_{i}}^{l_{\Gamma}(a)} c_{i, m} t^{m}$ with $c_{i, a_{i}} \neq 0$ for $i=1, \ldots, n$. Note that the coefficient of $t^{l_{\Gamma}(a)}$ in $g(\varphi(t))$ is equal to

$$
\begin{aligned}
\left.\frac{1}{l_{\Gamma}(a) !} \cdot \frac{d^{l_{\Gamma}(a)} g(\varphi(t))}{d t^{l_{\Gamma}(a)}}\right|_{t=0} & =\left.\frac{1}{l_{\Gamma}(a) !} \cdot \frac{d^{l_{\Gamma}(a)} g_{\gamma}(\varphi(t))}{d t^{l_{\Gamma}(a)}}\right|_{t=0} \\
& =g_{\gamma}\left(c_{1, a_{1}}, \ldots, c_{n, a_{n}}\right),
\end{aligned}
$$

which is nonzero for every $a$ in $\sigma_{\gamma, I}$ and $\left(c_{1, a_{1}}, \ldots, c_{n, a_{n}}\right)$ in $X_{\gamma}$. One deduces from this that $\mathscr{X}_{a, l_{\Gamma}(a)}(g)$ is isomorphic to $X_{\gamma} \times_{\kappa} \mathbb{A}_{\kappa}^{n l_{\Gamma}(a)-s(a)}$ via the map

$$
\varphi(t) \mapsto\left(\left(c_{i, a_{i}}\right)_{1 \leq i \leq n},\left(c_{i, m}\right)_{1 \leq i \leq n, a_{i}+1 \leq m \leq l_{\Gamma}(a)}\right) .
$$

Here the action of $\mathbb{G}$ on $\mathbb{A}_{\kappa}^{1}$ is trivial. For any $s$ in $\mathbb{G}$, the $\operatorname{arc} \varphi(s t)$ is mapped to

$$
\left(\left(s^{a_{i}} c_{i, a_{i}}\right)_{1 \leq i \leq n},\left(c_{i, m}\right)_{1 \leq i \leq n, a_{i}+1 \leq m \leq l_{\Gamma}(a)}\right),
$$

which is by definition equal to

$$
s \cdot\left(\left(c_{i, a_{i}}\right)_{1 \leq i \leq n},\left(c_{i, m}\right)_{1 \leq i \leq n, a_{i}+1 \leq m \leq l_{\Gamma}(a)}\right) .
$$


This means that the $\mathbb{G}$-action is compatible with the isomorphism; that is, the isomorphism is $\mathbb{G}$-equivariant. Then item (ii) follows.

Remark 4.2. We do not know yet how to compute $\left[\mathscr{L}_{a, l_{\Gamma}(a)+k}(g)\right]$ for $k \geq 0$ without the assumptions as in Lemma 4.1.

Remark 4.3. Lemma 4.1 and Remark 4.2 explain the reason why in the rest of this paper we will always assume that no vertex of the Newton polyhedron $\Gamma$ of $g$ lies in a coordinate plane; that is, $a_{i} \leq l_{\Gamma}(a)$ for any $i=1, \ldots, n$. In this case, $l_{\Gamma}(a)$ is expressed as $\sum_{i=1}^{n} \alpha_{i} a_{i}$ with $\alpha_{i}>0$ for any $i=1, \ldots, n$. By Lemma 4.1, this hypothesis guarantees that, for every compact face $\gamma$ of $\Gamma$, with $I$ in $\mathfrak{S}_{\gamma}$, all the terms of the sum $\sum_{a \in \sigma_{\gamma, I}}\left[\mathscr{L}_{a, l_{\Gamma}(a)}(g)\right]$ are nonzero if $\Phi_{\gamma, I}$ is nonzero, and all the terms of the sum $\sum_{a \in \sigma_{\gamma, I}}\left[\mathscr{Q}_{a, l_{\Gamma}(a)+k}(g)\right]$ (where $k>0$ ) are nonzero if $\Psi_{\gamma, I}$ is nonzero. In our work, we want to consider sums of this type that can be reduced to the case of Lemma 2.1 .

4D. An explicit formula for $i_{1}^{*} \mathscr{Y}_{\boldsymbol{g}}$. Assume that $g$ is a regular function on $\mathbb{A}_{\kappa}^{n}$ that is nondegenerate with respect to its Newton polyhedron $\Gamma$, that no vertex of $\Gamma$ lies in a coordinate $m$-plane $(m=1, \ldots, n-1)$, and that $X_{0}(g)$ contains $\mathbb{A}_{\kappa}^{n_{1}} \times{ }_{\kappa}\{0\}$. One then deduces from Remark 4.3 and Lemma 4.1 that

$$
i_{1}^{*} Z^{0}(T)=\sum_{\gamma \in \Gamma_{c}} \sum_{I \in \mathfrak{S}_{\gamma}} \sum_{a \in \sigma_{\gamma, I}} i_{1}^{*} \Phi_{\gamma, I} \mathbb{L}^{-s(a)} T^{l_{\Gamma}(a)},
$$

and that

$$
\begin{aligned}
& i_{1}^{*} Z^{1}(T)=i_{1}^{*}\left(\sum_{\gamma \in \Gamma_{c}} \sum_{I \in \mathfrak{S}_{\gamma}} \sum_{a \in \sigma_{\gamma, I}} \Psi_{\gamma, I} \mathbb{\mathbb { L }}^{-s(a)} T^{l_{\Gamma}(a)} \sum_{k \geq 1} \mathbb{L}^{-k} T^{k}\right) \\
& =\frac{\mathbb{Q}^{-1} T}{1-\mathbb{Q}^{-1} T} \sum_{\gamma \in \Gamma_{c}} \sum_{I \in \mathfrak{S}_{\gamma}} \sum_{a \in \sigma_{\gamma, I}} i_{1}^{*} \Psi_{\gamma, I^{\mathbb{Q}}}{ }^{-s(a)} T^{l_{\Gamma}(a)} .
\end{aligned}
$$

Proposition 4.4. With the previous notation and hypotheses, the following formula holds in $M_{\mathbb{A}_{\kappa}^{n_{1}} \times_{\kappa} \mathbb{G}}^{\mathbb{G}}$ :

$$
i_{1}^{*} \mathscr{S}_{g}=\sum_{\gamma \in \Gamma_{c}}(-1)^{n+1-\operatorname{dim}(\gamma)} \sum_{I \in \mathfrak{S}_{\gamma}}(-1)^{|I|}\left[\mathbb{A}_{\kappa}^{n_{1}} \times_{X_{0}(g)}\left(\Phi_{\gamma, I}-\Psi_{\gamma, I}\right)\right] .
$$

Proof. The positivity of the sum function $s$ on $\overline{\sigma_{\gamma, I}} \backslash\{0\}$ is evident, and that of the function $l_{\Gamma}$ on $\overline{\sigma_{\gamma, I}} \backslash\{0\}$ follows straightforward from Remark 4.3. Applying Lemma 2.1, notice that $\operatorname{dim}\left(\sigma_{\gamma, I}\right)=n-|I|-\operatorname{dim}(\gamma)$; we have

$$
\begin{aligned}
\lim _{T \rightarrow \infty} \sum_{a \in \sigma_{\gamma, I}} \Phi_{\gamma, I} \mathbb{L}^{-s(a)} T^{l_{\Gamma}(a)} & =\Phi_{\gamma, I} \lim _{T \rightarrow \infty} \sum_{a \in \sigma_{\gamma, I}} \mathbb{L}^{-s(a)} T^{l_{\Gamma}(a)} \\
& =(-1)^{n-|I|-\operatorname{dim}(\gamma)} \Phi_{\gamma, I}
\end{aligned}
$$


and

$$
\begin{aligned}
\lim _{T \rightarrow \infty} \sum_{a \in \sigma_{\gamma, I}} \Psi_{\gamma, I} \mathbb{L}^{-s(a)} T^{l_{\Gamma}(a)} & =\Psi_{\gamma, I} \lim _{T \rightarrow \infty} \sum_{a \in \sigma_{\gamma, I}} \mathbb{Q}^{-s(a)} T^{l_{\Gamma}(a)} \\
& =(-1)^{n-|I|-\operatorname{dim}(\gamma)} \Psi_{\gamma, I} .
\end{aligned}
$$

It follows that

$$
\lim _{T \rightarrow \infty} i_{1}^{*} Z^{0}(T)=\sum_{\gamma \in \Gamma_{c}}(-1)^{n-\operatorname{dim}(\gamma)} \sum_{I \in \mathfrak{S}_{\gamma}}(-1)^{|I|} i_{1}^{*} \Phi_{\gamma, I},
$$

and

$$
\lim _{T \rightarrow \infty} i_{1}^{*} Z^{1}(T)=\sum_{\gamma \in \Gamma_{c}}(-1)^{n+1-\operatorname{dim}(\gamma)} \sum_{I \in \mathfrak{S}_{\gamma}}(-1)^{|I|} i_{1}^{*} \Psi_{\gamma, I}
$$

Then the proposition is proved.

Example 4.5 (cf. Example 3.5). In the case where $\Gamma_{g}$ has a unique compact face $P$, the classes $\Psi_{P, I}$ vanish. If we assume that $\alpha_{i}>0$ for every $i=1, \ldots, n$, we have

$$
i_{1}^{*} \mathscr{Y}_{g}=(-1)^{n+1} \sum_{I \subset\left\{1, \ldots, n_{1}\right\}}(-1)^{|I|}\left[\mathbb{A}_{\kappa}^{n_{1}} \times_{X_{0}(g)} \Phi_{P, I}\right] .
$$

Corollary 4.6 [Guibert 2002]. Assume that $g$ is given by $g(x)=\sum_{\alpha \in \mathbb{N}_{>0}^{n}} a_{\alpha} x^{\alpha}$ in $\kappa[x]$ with $g(0)=0$. If $g$ is nondegenerate with respect to $\Gamma$, then

$$
\mathscr{Y}_{g, 0}=(-1)^{n-1} \sum_{\gamma \in \Gamma_{c}}(-1)^{\operatorname{dim}(\gamma)}\left[\{0\} \times_{X_{0}(g)}\left(\Phi_{\gamma, I}-\Psi_{\gamma, I}\right)\right]
$$

holds in $\mathcal{M}_{\mathbb{G}}^{\mathbb{G}}$.

Proof. (See Remark 3.6) Apply Proposition 4.4 to the case $n_{1}=0$. Here the natural inclusion $i_{1}: \mathbb{A}_{\kappa}^{n_{1}} \hookrightarrow \mathbb{A}_{\kappa}^{n}$ reduces to the inclusion $i_{0}:\{0\} \hookrightarrow \mathbb{A}_{\kappa}^{n}$. Moreover, in this case, by Lemma 3.3, for every compact face $\gamma$ of $\Gamma$, we have $\mathfrak{S}_{\gamma}=\{\varnothing\}$. Thus this corollary follows. Observe that this formula was already obtained by Guibert (cf. [2002, Proposition 2.1.6]).

4E. Consider the function $g(x)=\sum_{\alpha \in H \cap \mathbb{N}^{n}} a_{\alpha} x^{\alpha}$ on $\mathbb{A}_{\kappa}^{n}$, where $H$ is the hyperplane in $\mathbb{R}_{\geq 0}^{n}$ defined by the equation

$$
\alpha_{1}+\cdots+\alpha_{n_{1}}=\alpha_{n_{1}+1}+\cdots+\alpha_{p},
$$

for some fixed $p$ such that $n_{1}<p \leq n$. Here, as well as in Corollary 4.6, we use the notation $x^{\alpha}$ for $x_{1}^{\alpha_{1}} \cdots x_{n}^{\alpha_{n}}$, where $\alpha=\left(\alpha_{1}, \ldots, \alpha_{n}\right)$. Because $\operatorname{supp}(g)$ lies on the hyperplane $H$, the compact faces of $\Gamma$ are contained in $H$. Moreover, for the same reason, for each compact $\gamma$, there exist noncompact faces of $\Gamma$ leaning on $\gamma$. Note that, in this case, $\mathbb{A}_{\kappa}^{n_{1}}$ is naturally viewed as a subset of $X_{0}(g)$. 
Lemma 4.7. Assume that $g(x)=\sum_{\alpha \in H \cap \mathbb{N}^{n}} a_{\alpha} x^{\alpha}$ is nondegenerate with respect to $\Gamma$. Then, for every compact face $\gamma$ of $\Gamma$, we have $\left|\mathfrak{M}_{\gamma}\right|=1$, and the unique element of $\mathfrak{M}_{\gamma}$ is nonempty.

Proof. Let $\gamma$ be a compact face of $\Gamma$. Assume that $\gamma+\mathbb{R}_{\geq 0}^{I}$ is a face of $\Gamma$. Then, by Lemma 3.3, the cone $\sigma_{\gamma, I}$ is contained in $\mathbb{R}_{\geq 0}^{n_{1}} \times \mathbb{R}_{>0}^{n_{2}}$ if and only if $I$ is contained in $\left\{1, \ldots, n_{1}\right\}$. Furthermore, we claim that if $\gamma+\mathbb{R}_{\geq 0}^{I}$ and $\gamma+\mathbb{R}_{\geq 0}^{J}$ are faces leaning on $\gamma$ such that the corresponding cones $\sigma_{\gamma, I}$ and $\sigma_{\gamma, J}$ are both contained in $\mathbb{R}_{\geq 0}^{n_{1}} \times \mathbb{R}_{>0}^{n_{2}}$, then so is $\gamma+\mathbb{R}_{\geq 0}^{I \cup J}$. Indeed, since $\left(\alpha_{1}, \ldots, \alpha_{n}\right)$ is in $H$, one deduces that if $I$ and $J$ are contained in $\left\{1, \ldots, n_{1}\right\}$, the intersection of $\gamma+\mathbb{R}_{>0}^{I \cup J}$ with the interior of $\Gamma$ is empty. This, together with the fact that $\gamma+\mathbb{R}_{\geq 0}^{I}$ and $\gamma+\mathbb{R}_{\geq 0}^{J}$ are faces of $\Gamma$, shows that $\gamma+\mathbb{R}_{\geq 0}^{I \cup J}$ is a face of $\Gamma$ leaning on $\gamma$ such that $\sigma_{\gamma, I \cup J}$ is contained in $\mathbb{R}_{\geq 0}^{n_{1}} \times \mathbb{R}_{>0}^{n_{2}}$.

As a consequence of the above claim, for each compact face $\gamma$ of $\Gamma$, there exists a unique maximal subset $M$ of $\left\{1, \ldots, n_{1}\right\}$ such that $\gamma+\mathbb{R}_{\geq 0}^{M}$ is a face of $\Gamma$ that leans on $\gamma$, and $\sigma_{\gamma, M}$ is contained in $\mathbb{R}_{\geq 0}^{n_{1}} \times \mathbb{R}_{>0}^{n_{2}}$. The nonemptiness of the set $M$ follows from the fact that $\operatorname{supp}(g)$ lies on the hyperplane $H$.

Proposition 4.8. Assume that $g(x)=\sum_{\alpha \in H \cap \mathbb{N}_{>0}^{n}} a_{\alpha} x^{\alpha}$ is nondegenerate with respect to $\Gamma$. Then $\int_{\mathbb{A}_{\kappa}} i_{1}^{*} \mathscr{S}_{g}$ vanishes in $M_{\mathbb{G}}^{\mathbb{G}}$.

Proof. Let $\gamma$ be a compact face of $\Gamma$. By Lemma 4.7, the set $\mathfrak{M}_{\gamma}$ has a unique element and this element is nonempty. Assume $\mathfrak{M}_{\gamma}=\{M\}$ with $|M| \geq 1$. Note that $A_{\gamma}=\int_{\mathbb{A}_{\kappa} d_{1}} i_{1}^{*} \Phi_{\gamma, I}$ and $B_{\gamma}=\int_{\mathbb{A}_{\kappa} d_{1}} i_{1}^{*} \Psi_{\gamma, I}$ depend only on $\gamma$, not on $I$ contained in $M$. Because $\sum_{j=0}^{m}(-1)^{j}\left(\begin{array}{c}m \\ j\end{array}\right)=0$ for $m \geq 1$, one deduces that

$$
\sum_{I \subset M}(-1)^{|I|}\left(A_{\gamma}-B_{\gamma}\right)=0 .
$$

The hypothesis on $g$ that $\alpha \in H \cap \mathbb{N}_{>0}^{n}$ means no vertex of the Newton polyhedron $\Gamma$ of $g$ lies in a coordinate plane. By Proposition 4.4, the image $\int_{A_{k}} i_{1}^{*} \mathscr{Y}_{g}$ of $\mathscr{Y}_{g}$ vanishes in $\mu_{\mathbb{G}}^{\mathbb{G}}$.

\section{The Kontsevich-Soibelman conjecture}

In this section, we will show that under certain assumptions, Conjecture 1.1 is true.

5A. Composition with a polynomial in two variables. We consider Conjecture 1.1 of Kontsevich and Soibelman in the case where $F$ has the form $F(x, y, z)=$ $f\left(g_{1}(x, y), g_{2}(z)\right)$, where $f$ is a polynomial in two variables with $f(0, y)$ nonzero of positive degree, $g_{1}$ is a function on $\mathbb{A}_{\kappa}^{d_{1}} \times{ }_{\kappa} \mathbb{A}_{\kappa}^{d_{2}}$ such that $g_{1}\left(t x, t^{-1} y\right)=g_{1}(x, y)$ and $g_{1}(0,0)=0$, and $g_{2}$ is a regular function on $\mathbb{A}_{\kappa}^{d_{3}}$. Let $\mathbf{g}=g_{1} \times g_{2}$ and $X_{0}(\mathbf{g})=\left\{(x, y, z) \mid g_{1}(x, y)=g_{2}(z)=0\right\}$. In particular, $X_{0}(\mathbf{g})$ contains $\mathbb{A}_{k}^{d_{1}} \times\{0\}$. 
We denote by $i_{1}$ the inclusion of $\mathbb{A}_{\kappa}^{d_{1}} \times{ }_{\kappa} \mathbb{G}$ into $X_{0}(f \circ \mathbf{g}) \times_{\kappa} \mathbb{G}$. Recall that, in this case, $h(z)=f\left(0, g_{2}(z)\right)$.

Theorem 5.1. Assume that $f$ is a polynomial in two variables with $f(0, y)$ nonzero of positive degree. Let $g_{1}$ be a regular function on $\mathbb{A}_{\kappa}^{d_{1}} \times{ }_{\kappa} \mathbb{A}_{\kappa}^{d_{2}}$ nondegenerate with respect to its Newton polyhedron $\Gamma_{g_{1}}$ such that $g_{1}(0,0)=0$, no vertex of $\Gamma_{g_{1}}$ lies in a coordinate plane, and $g_{1}\left(t x, t^{-1} y\right)=g_{1}(x, y)$ for every $t$ in $\mathbb{G}$. Let $g_{2}$ be a regular function on $\mathbb{A}_{\kappa}^{d_{3}}$. Then, the formula

$$
\int_{\mathbb{A}_{\kappa}^{d_{1}}} i_{1}^{*} \mathscr{Y}_{f \circ \mathbf{g}}=\mathbb{L}^{d_{1}} \mathscr{Y}_{h, 0}
$$

holds in $M_{\mathbb{G}}^{\mathbb{G}}$. In other words, in this case, Conjecture 1.1 is true.

Proof. In [Guibert et al. 2009], Guibert, Loeser and Merle consider the motivic Milnor fiber of a composition of the form $f\left(g_{1}, g_{2}\right)$ where $g_{1}$ and $g_{2}$ have no variable in common and $f$ is a polynomial in $\kappa[x, y]$ such that $f(0, y)$ is nonzero of positive degree. To describe it, they used the generalized convolution operators $\Psi_{Q}$ defined in [Guibert et al. 2005] and the tree of contact $\tau(f, 0)$ constructed in terms of Puiseux expansions by Guibert [2002]. Here 0 is the origin of $\mathbb{A}_{\kappa}^{d}$, with $d=d_{1}+d_{2}+d_{3}$. To any rupture vertex $v$ of $\tau(f, 0)$ one attaches a weighted homogeneous polynomial $Q_{v}$ in $\kappa[X, Y]$. The virtual objects $A_{v}$ are defined inductively in terms of the tree of contact $\tau(f, 0)$ and $A_{v_{0}}$, where $v_{0}$ is the first (extended) rupture vertex of the tree and $A_{v_{0}}$ depends only on $g$. Let $i$ be the inclusion of $X_{0}(\mathbf{g}) \times_{\kappa} \mathbb{G}$ into $X_{0}(f \circ \mathbf{g}) \times_{\kappa} \mathbb{G}$. Let $m_{0}$ be the order of 0 as a root of $f(0, y)$. By the main theorem of [Guibert et al. 2009], the formula

$$
i^{*} \mathscr{Y}_{f \circ \mathbf{g}}=\mathscr{Y}_{g_{2} m_{0}}\left(\left[X_{0}\left(g_{1}\right)\right]\right)-\sum_{v} \Psi_{Q_{v}}\left(A_{v}\right)
$$

holds in $M_{X_{0}(\mathbf{g}) \times_{\kappa} \mathbb{G}}^{\mathbb{G}}$, where $\Psi_{Q_{v}}$ denotes the convolution defined in the same paper and the sum runs over the augmented set of rupture vertices of the tree $\tau(f, 0)$. The $i_{1}$ in the theorem is the inclusion of $\mathbb{A}_{\kappa}^{d_{1}} \times{ }_{\kappa} \mathbb{G}$ into $X_{0}(f \circ \mathbf{g}) \times_{\kappa} \mathbb{G}$, but by abuse of notation, we also use $i_{1}$ for the inclusion $\mathbb{A}_{\kappa}^{d_{1}} \times{ }_{\kappa} \mathbb{G} \hookrightarrow X_{0}(\mathbf{g}) \times_{\kappa} \mathbb{G}$. Thus $i_{1}$ and $i \circ i_{1}$ are in fact the same thing. Applying the operator $\int_{\mathbb{A}_{\kappa}^{d_{1}}} i_{1}^{*}$ to both sides of the previous formula, we have

$$
\int_{\mathbb{A}_{\kappa}^{d_{1}}} i_{1}^{*} \varphi_{f \circ \mathbf{g}}=\int_{\mathbb{A}_{\kappa}^{d_{1}}} i_{1}^{*} \varphi_{g_{2} m_{0}}\left(\left[X_{0}\left(g_{1}\right)\right]\right)-\sum_{v} \int_{\mathbb{A}_{\kappa}^{d_{1}}} i_{1}^{*} \Psi_{Q_{v}}\left(A_{v}\right) .
$$

We claim that, with the previous notation and hypotheses, the formula

$$
\int_{\mathbb{A}_{\kappa}^{d_{1}}} i_{1}^{*} \mathscr{Y}_{g_{2} m_{0}}\left(\left[X_{0}\left(g_{1}\right)\right]\right)=\mathbb{L}^{d_{1}} \mathscr{S}_{h, 0}
$$


holds in $\mathcal{M}_{\mathbb{G}}^{\mathbb{G}}$. Indeed, as in the proof of [Guibert et al. 2006, Theorem 5.18], one can check that

$$
i^{*} \mathscr{Y}_{g_{2} m_{0}}\left(\left[X_{0}\left(g_{1}\right)\right]\right)=\left[g_{1}^{-1}(0)\right] \otimes \mathscr{Y}_{g_{2}}^{m_{0}} .
$$

By the hypotheses on $g_{1}$ and the fact that $i_{1}\left(\mathbb{A}_{\kappa}^{d_{1}}\right) \cap g_{2}^{-1}(0)=\{0\}$, we have

$$
i_{1}^{*}\left[g_{1}^{-1}(0)\right]=\left[\mathbb{A}_{\kappa}^{d_{1}}\right]=\mathbb{L}^{d_{1}} \text { and } i_{1}^{*} \mathscr{S}_{g_{2} m_{0}}=i_{0}^{*} \mathscr{Y}_{g_{2} m_{0}}=\mathscr{Y}_{g_{2}{ }^{m_{0}}, 0} .
$$

One deduces that

$$
i_{1}^{*} \mathscr{Y}_{g_{2} m_{0}}\left(\left[X_{0}\left(g_{1}\right)\right]\right)=i_{1}^{*}\left(\left[g_{1}^{-1}(0)\right] \bigotimes \mathscr{Y}_{g_{2} m_{0}}\right)=\mathbb{L}^{d_{1}} \mathscr{Y}_{g_{2}{ }^{m}, 0} .
$$

By the definitions of $h$ and $m_{0}$, we have $\mathscr{S}_{g_{2}{ }^{m_{0}}, 0}=\mathscr{S}_{h, 0}$, and the claim then follows. So, in order to finish the proof of Theorem 5.1, it suffices to prove that $\int_{\AA_{\kappa}^{d_{1}}} i_{1}^{*} \Psi_{Q_{v}}\left(A_{v}\right)=0$ for every (extended) rupture vertex $v$ of $\tau(f, 0)$.

Let $v_{0}$ be the first (extended) rupture vertex of the tree of contact $\tau(f, p)$. As in [Guibert et al. 2009], the virtual object $A_{v_{0}}$ in

$$
\mathcal{M}_{X_{0}(\mathbf{G}) \times_{\kappa}\left(\mathbb{A}_{\kappa}^{1} \times{ }_{\kappa} \mathbb{G}\right)}^{\mathbb{G}}
$$

is defined by $A_{v_{0}}:=\mathscr{Y}_{g_{2}}^{\prime} \otimes \mathscr{S}_{g_{1}}$, where $\mathscr{Y}_{g_{2}}^{\prime}$ is an element in $\mathcal{M}_{X_{0}\left(g_{2}\right) \times_{\kappa} A_{\kappa}^{1}}^{\mathbb{G}}$ that is the "disjoint sum" of $\mathscr{Y}_{g_{2}}$ in $M_{X_{0}\left(g_{2}\right) \times_{k} \mathbb{G}}^{\mathbb{G}}$ and $X_{0}\left(g_{2}\right)$ in $M_{X_{0}\left(g_{2}\right)}$.

Lemma 5.2. Assume that $g_{1}$ is a regular function on $\mathbb{A}_{\kappa}^{d_{1}} \times{ }_{\kappa} \mathbb{A}_{\kappa}^{d_{2}}$ nondegenerate with respect to its Newton polyhedron $\Gamma_{g_{1}}$ such that $g_{1}(0,0)=0$, no vertex of $\Gamma_{g_{1}}$ lies in a coordinate plane, and $g_{1}\left(t x, t^{-1} y\right)=g_{1}(x, y)$ for every $t$ in $\mathbb{G}$. Let $g_{2}$ be a regular function on $\mathbb{A}_{\kappa}^{d_{3}}$. Then $\int_{\mathbb{A}_{\kappa}^{d_{1}}} i_{1}^{*} \Psi_{Q}\left(A_{v_{0}}\right)$ vanishes in $M_{\mathbb{G}}^{\mathbb{G}}$ for every quasihomogeneous polynomial $Q$.

Proof. The assumptions on $g_{1}$ mean that we can write $g_{1}$ in the form

$$
g_{1}(x, y)=\sum_{(\alpha, \beta) \in H \cap \mathbb{N}_{>0}^{d_{1}+d_{2}}} a_{\alpha \beta} x_{1}^{\alpha_{1}} \cdots x_{d_{1}}^{\alpha_{d_{1}}} y_{1}^{\beta_{1}} \cdots y_{d_{2}}^{\beta_{d_{2}}},
$$

where $H$ is given by $\alpha_{1}+\cdots+\alpha_{d_{1}}=\beta_{1}+\cdots+\beta_{d_{2}}$. By Proposition 4.8, $\int_{\mathbb{A}_{k}} i_{1}^{*} \mathscr{Y}_{g_{1}}$ vanishes in $\mathcal{M}_{\mathbb{G}}^{\mathbb{G}}$, hence $\int_{\mathbb{A}_{\kappa}^{d_{1}}} i_{1}^{*} A_{v_{0}}$ vanishes in $M_{\mathbb{A}_{\kappa}^{1} \times_{\kappa} \mathbb{G}}^{\mathbb{G}}$. Here $i_{1}$ is once again abused to denote the natural inclusion $\mathbb{A}_{\kappa}^{d_{1}} \times{ }_{\kappa} \mathbb{A}_{\kappa}^{1} \times{ }_{\kappa} \mathbb{G} \hookrightarrow X_{0}(\mathbf{g}) \times{ }_{\kappa} \mathbb{A}_{\kappa}^{1} \times_{\kappa} \mathbb{G}$. Because the diagram

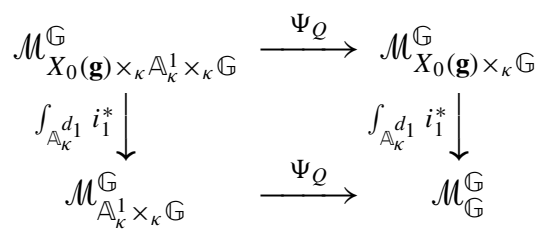

commutes, the lemma follows. 
Let $v$ be an arbitrary rupture vertex of the tree of contact $\tau(f, 0)$ and $a(v)$ the predecessor of $v$ in the augmented set of rupture vertices. Then the polynomial $Q_{v}$ is a factor of $Q_{a(v)}$. Suppose that $Q_{v}(X, 1)$ has $m_{v}$ disjoint zeroes in $\mathbb{A}_{\kappa}^{1}$.

Lemma 5.3. The equality $A_{v}=m_{v} A_{a(v)}$ holds in $M_{X_{0}(\mathbf{g}) \times_{\kappa} A_{\kappa}^{1} \times_{\kappa} \mathbb{G}}^{\mathbb{G}}$.

Proof. We first notice that $Q_{v}^{-1}(0)$ is a smooth subvariety in $\mathbb{G} \times{ }_{\kappa} \mathbb{G}$ equivariant under a diagonal $\mathbb{G}$-action, and that the second projection $\mathrm{pr}_{2}$ of the product $\mathbb{A}_{\kappa}^{1} \times{ }_{\kappa} \mathbb{G}$ induces a homogeneous fibration $Q_{v}^{-1}(0) \rightarrow \mathbb{G}$. We denote by $B_{v}$ the restriction of $A_{a(v)}$ above $Q_{v}^{-1}(0)$. Then, by [Guibert et al. 2009], the element $A_{v}$ in $M_{X_{0}(\mathbf{g}) \times{ }_{\kappa} A_{\kappa}^{1} \times{ }_{\kappa} \mathbb{G}}^{\mathbb{G}}$ is defined as the external product of the class of id: $\mathbb{A}_{\kappa}^{1} \rightarrow \mathbb{A}_{\kappa}^{1}$ by the induced map $\mathrm{pr}_{2}: B_{v} \rightarrow \mathbb{G}$, which is diagonally monomial when restricted to $X_{0}(\mathbf{g}) \times{ }_{\kappa} \mathbb{G} \times{ }_{\kappa} \mathbb{G}$.

Consider the fibration $\mathrm{pr}_{2}: B_{v} \rightarrow \mathbb{G}$ defined by the composition of $B_{v} \rightarrow Q_{v}^{-1}(0)$ and $\mathrm{pr}_{2}: Q_{v}^{-1}(0) \rightarrow \mathbb{G}$. Then each fiber of $\mathrm{pr}_{2}: B_{v} \rightarrow \mathbb{G}$ is a disjoint union of $m_{v}$ copies of a fiber of $A_{a(v)} \rightarrow \mathbb{A}_{\kappa}^{1} \times{ }_{\kappa} \mathbb{G}$ over one point $(a, b)$ in $\mathbb{A}_{\kappa}^{1} \times{ }_{\kappa} \mathbb{G}$. It follows that $A_{v}=m_{v} A_{a(v)}$ in $M_{X_{0}(\mathbf{g}) \times_{\kappa}\left(\mathbb{A}_{\kappa}^{1} \times_{\kappa} \mathbb{G}\right)}^{\mathbb{G}}$.

It follows from Lemma 5.2 and Lemma 5.3 that $\int_{A_{k} d_{1}} i_{1}^{*} \Psi_{Q_{v}}\left(A_{v}\right)=0$ for every (extended) rupture vertex $v$ of $\tau(f, 0)$, completing the proof of Theorem 5.1.

Remark 5.4. In the case $f(x, y)=x+y$, the result can also be obtained directly from the motivic Thom-Sebastiani theorem [Denef and Loeser 1999b; 2001].

5B. In the next proposition, we prove the conjecture of Kontsevich and Soibelman under some other conditions on $F=g$, namely assuming $F$ is nondegenerate with respect to its Newton polyhedron $\Gamma$ and no vertex of $\Gamma$ lies in a coordinate plane.

Proposition 5.5. Let $g$ be a regular function on $\mathbb{A}_{\kappa}^{d_{1}} \times{ }_{\kappa} \mathbb{A}_{\kappa}^{d_{2}} \times{ }_{\kappa} \mathbb{A}_{\kappa}^{d_{3}}$ such that $g(0,0, z)=0$ for every $z$ in $\mathbb{A}_{\kappa}^{d_{3}}, g\left(t x, t^{-1} y, z\right)=g(x, y, z)$ for every $t$ in $\mathbb{G}$, and $(x, y, z)$ in $\mathbb{A}_{\kappa}^{d_{1}} \times{ }_{\kappa} \mathbb{A}_{\kappa}^{d_{2}} \times{ }_{\kappa} \mathbb{A}_{\kappa}^{d_{3}}$. If $g$ is nondegenerate with respect to its Newton polyhedron $\Gamma$ and no vertex of $\Gamma$ lies in a coordinate plane, then $\int_{\mathbb{A}_{\kappa}} i_{1}^{*} \mathscr{\varphi}_{g}$ vanishes in $\mathcal{M}_{\mathbb{G}}^{\mathbb{G}}$. In other words, Conjecture 1.1 is true in this case.

Proof. Write the function $g$ in the form

$$
g(x, y, z)=\sum_{(a, b, c) \in H \cap \mathbb{N}_{>0}^{d}} g_{a, b, c} x^{a} y^{b} z^{c},
$$

where $d=d_{1}+d_{2}+d_{3}$ and $H$ is given by the equation $a_{1}+\cdots+a_{d_{1}}=b_{1}+\cdots+b_{d_{2}}$. By Proposition 4.8, $\int_{\mathbb{A}_{\kappa}^{d_{1}}} i_{1}^{*} \mathscr{S}_{g}$ vanishes in $\mathcal{M}_{\mathbb{G}}^{\mathbb{G}}$. Notice that in this case $h(z)=$ $F(0,0, z)=g(0,0, z)=0$, hence $\mathscr{S}_{h, 0}$ also vanishes in $M_{\mathbb{G}}^{\mathbb{G}}$. 
5C. Functions of Steenbrink type. We consider now the case that

$$
F(x, y, z)=g(x, y, z)+h(z)^{\ell},
$$

where $g$ is as in Proposition 5.5, $h(z)$ is regular on $\mathbb{A}_{\kappa}^{d_{3}}$ such that $h(0)=0$, and $\ell$ is a large enough natural number. By composition with the projection, we will view $h$ as a function on $\mathbb{A}_{\kappa}^{d}$.

Theorem 5.6. Let $F(x, y, z)=g(x, y, z)+h(z)^{\ell}$, where $g$ is as in Proposition 5.5, $h(z)$ is regular on $\mathbb{A}_{\kappa}^{d_{3}}$ such that $h(0)=0$, and $\ell$ is a natural number. There exists a positive real number $N$ such that, ife $>N$, the following formula holds in $M_{\mathbb{G}}^{\mathbb{G}}$ :

$$
\int_{\mathbb{A}_{\kappa}^{d_{1}}} i_{1}^{*} \mathscr{S}_{F}=\mathbb{L}^{d_{1}} \mathscr{Y}_{h^{\ell}, 0} .
$$

Proof. Let us denote by $i$ and $j$ the inclusions of $\left(X_{0}(g) \cap X_{0}(h)\right) \times{ }_{\kappa} \mathbb{G}$ into $X_{0}(g) \times_{\kappa} \mathbb{G}$ and $X_{0}(F) \times_{\kappa} \mathbb{G}$, respectively. The existence of $N$ is shown by [Guibert et al. 2006, Theorem 5.7]. Also by this theorem, for $\ell>N$, we have

$$
j^{*} \mathscr{Y}_{F}-i^{*} \mathscr{S}_{g}=\mathscr{Y}_{h^{\ell}}\left(\left[X_{0}(g)\right]\right)-\Psi_{\Sigma}\left(\mathscr{Y}_{h^{\ell}}\left(\mathscr{Y}_{g}\right)\right),
$$

where $\Psi_{\Sigma}$ is the convolution defined in [Guibert et al. 2006]. Then we get

$$
\int_{\mathbb{A}_{\kappa}^{d_{1}}} i_{1}^{*} \mathscr{G}_{F}-\int_{\mathbb{A}_{\kappa}^{d_{1}}} i_{1}^{*} \mathscr{Y}_{g}=\int_{\mathbb{A}_{\kappa}^{d_{1}}} i_{1}^{*} \mathscr{S}_{h^{\ell}}\left(\left[X_{0}(g)\right]\right)-\int_{\mathbb{A}_{\kappa}^{d_{1}}} i_{1}^{*} \Psi_{\Sigma}\left(\mathscr{Y}_{h^{\ell}}\left(\mathscr{Y}_{g}\right)\right) .
$$

Now, by Proposition 5.5, $\int_{A_{1}} i_{1}^{*} \mathscr{Y}_{g}=0$. An analogue to the proof of Lemma 5.2 shows that $\int_{A_{\kappa}^{d_{1}}} i_{1}^{*} \Psi_{\Sigma}\left(\mathscr{Y}_{h^{\ell}}\left(\mathscr{S}_{g}\right)\right)$ vanishes. One deduces that

$$
\int_{\mathbb{A}_{\kappa}^{d_{1}}} i_{1}^{*} \mathscr{Y}_{F}=\int_{\mathbb{A}_{\kappa}^{d_{1}}} i_{1}^{*} \mathscr{Y}_{h^{\ell}}\left(\left[X_{0}(g)\right]\right) .
$$

Define a function $g^{\prime}$ on $\mathbb{A}_{\kappa}^{d_{1}} \times_{\kappa} \mathbb{A}_{\kappa}^{d_{2}}$ by setting $g^{\prime}(x, y)=g(x, y, 0)$. Then we have that $g^{\prime}(0,0)=0$ and $g^{\prime}\left(t x, t^{-1} y\right)=g^{\prime}(x, y)$ for any $t$ in $\mathbb{G}$. Furthermore, we have an identity in $M_{X_{0}(g)}$ as follows

$$
\left[X_{0}(g)\right]=\left[X_{0}\left(g^{\prime}\right)\right]+\left[\left\{(x, y, z) \in \mathbb{A}_{\kappa}^{d_{1}+d_{2}} \times\left(\mathbb{A}_{\kappa}^{d_{3}} \backslash\{0\}\right) \mid g(x, y, z)=0\right\}\right] .
$$

As in the proof of Theorem 5.1, since $h^{\ell}$ and $g^{\prime}$ have no variable in common, we have

$$
i_{1}^{*} \mathscr{S}_{h^{\ell}}\left(\left[X_{0}\left(g^{\prime}\right)\right]\right)=\mathbb{L}^{d_{1}} \mathscr{Y}_{h^{\ell}, 0}
$$

in $M_{A_{\kappa}^{d_{1}} \times \mathbb{G}}^{\mathbb{G}}$. It remains to notice that

$$
i_{1}^{*} \mathscr{S}_{h^{\ell}}\left(\left[\left\{(x, y, z) \in \mathbb{A}_{\kappa}^{d_{1}+d_{2}} \times\left(\mathbb{A}_{\kappa}^{d_{3}} \backslash\{0\}\right) \mid g(x, y, z)=0\right\}\right]\right)=0,
$$

because the intersection

$$
i_{1}\left(\mathbb{A}_{\kappa}^{d_{1}}\right) \cap\left\{(x, y, z) \in \mathbb{A}_{\kappa}^{d_{1}+d_{2}} \times\left(\mathbb{A}_{\kappa}^{d_{3}} \backslash\{0\}\right) \mid g(x, y, z)=0\right\}
$$


is empty. Thus, $\int_{\mathbb{A}_{\kappa}^{d_{1}}} i_{1}^{*} \mathscr{S}_{F}=\mathbb{L}^{d_{1}} \mathscr{S}_{h^{\ell}, 0}$ in $\mathcal{M}_{\mathbb{G}}^{\mathbb{G}}$, as needed.

\section{Acknowledgments}

This work was suggested by François Loeser, my advisor, who proposed that I consider the conjecture first in the case of composition $f\left(g_{1}, g_{2}\right)$ and encouraged me in each step of proof. I am deeply grateful to him for his suggestions of methods to approach the problem and for his help in preparing the manuscript. I would like to thank the referee for his contributions to the paper, which made it more readable.

\section{References}

[Denef and Loeser 1998] J. Denef and F. Loeser, "Motivic Igusa zeta functions", J. Algebraic Geom. 7:3 (1998), 505-537. MR 99j:14021 Zbl 0943.14010

[Denef and Loeser 1999a] J. Denef and F. Loeser, "Germs of arcs on singular algebraic varieties and motivic integration”, Invent. Math. 135:1 (1999), 201-232. MR 99k:14002 Zbl 0928.14004

[Denef and Loeser 1999b] J. Denef and F. Loeser, "Motivic exponential integrals and a motivic Thom-Sebastiani theorem”, Duke Math. J. 99 (1999), 285-309. MR 2000k:14006 Zbl 0966.14015

[Denef and Loeser 2001] J. Denef and F. Loeser, "Geometry on arc spaces of algebraic varieties", pp. 327-348 in European Congress of Mathematics (Barcelona, 2000), vol. I, edited by C. Casacuberta et al., Progr. Math. 201, Birkhäuser, Basel, 2001. MR 2004c:14037 Zbl 1079.14003

[Guibert 2002] G. Guibert, "Espaces d'arcs et invariants d'Alexander", Comment. Math. Helv. 77:4 (2002), 783-820. MR 2003k:14021 Zbl 1046.14008

[Guibert et al. 2005] G. Guibert, F. Loeser, and M. Merle, "Nearby cycles and composition with a nondegenerate polynomial”, Int. Math. Res. Not. 2005:31 (2005), 1873-1888. MR 2006f:11145 Zbl 1093.14032

[Guibert et al. 2006] G. Guibert, F. Loeser, and M. Merle, "Iterated vanishing cycles, convolution, and a motivic analogue of a conjecture of Steenbrink", Duke Math. J. 132:3 (2006), 409-457. MR 2007e:14011 Zbl 1173.14301

[Guibert et al. 2009] G. Guibert, F. Loeser, and M. Merle, "Composition with a two variable function”, Math. Res. Lett. 16 (2009), 439-448. MR 2010j:14021 Zbl 1187.14046

[Kontsevich and Soibelman 2008] M. Kontsevich and Y. Soibelman, "Stability structures, motivic Donaldson-Thomas invariants and cluster transformations", preprint, 2008. arXiv 0811.2435v1

[Toën 2006] B. Toën, "Derived Hall algebras", Duke Math. J. 135:3 (2006), 587-615. MR 2007h: 18021 Zbl 1117.18011

Communicated by Ehud Hrushovski

Received 2010-10-01 Revised 2010-12-06 Accepted 2011-01-19

leqthuong@math.jussieu.fr École Normale Supérieure, Départment de Mathématiques et Applications, UMR 8553 CNRS, 45 rue d'Ulm, 75230 Paris cedex 05, France

Current address:

Institut de Mathématiques de Jussieu, UMR 7586 CNRS, 4 place Jussieu, 75005 Paris, France 


\section{Algebra \& Number Theory}

msp.berkeley.edu/ant

\section{EDITORS}

MANAGING EDITOR

Bjorn Poonen

Massachusetts Institute of Technology

Cambridge, USA

\author{
EDITORIAL BOARD CHAIR \\ David Eisenbud \\ University of California \\ Berkeley, USA
}

\section{BOARD OF EDITORS}

Georgia Benkart

Dave Benson

Richard E. Borcherds

John H. Coates

J-L. Colliot-Thélène

Brian D. Conrad

Hélène Esnault

Hubert Flenner

Edward Frenkel

Andrew Granville

Joseph Gubeladze

Ehud Hrushovski

Craig Huneke

Mikhail Kapranov

Yujiro Kawamata

János Kollár

Yuri Manin

Barry Mazur

Philippe Michel

Susan Montgomery
University of Wisconsin, Madison, USA

University of Aberdeen, Scotland

University of California, Berkeley, USA

University of Cambridge, UK

CNRS, Université Paris-Sud, France

University of Michigan, USA

Universität Duisburg-Essen, Germany

Ruhr-Universität, Germany

University of California, Berkeley, USA

Université de Montréal, Canada

San Francisco State University, USA

Hebrew University, Israel

University of Kansas, USA

Yale University, USA

University of Tokyo, Japan

Princeton University, USA

Northwestern University, USA

Harvard University, USA

École Polytechnique Fédérale de Lausanne

University of Southern California, USA
Shigefumi Mori

Raman Parimala

Jonathan Pila

Victor Reiner

Karl Rubin

Peter Sarnak

Joseph H. Silverman

Michael Singer

Ronald Solomon

Vasudevan Srinivas

J. Toby Stafford

Bernd Sturmfels

Richard Taylor

Ravi Vakil

Michel van den Bergh

Marie-France Vignéras

Kei-Ichi Watanabe

Andrei Zelevinsky

Efim Zelmanov
RIMS, Kyoto University, Japan

Emory University, USA

University of Oxford, UK

University of Minnesota, USA

University of California, Irvine, USA

Princeton University, USA

Brown University, USA

North Carolina State University, USA

Ohio State University, USA

Tata Inst. of Fund. Research, India

University of Michigan, USA

University of California, Berkeley, USA

Harvard University, USA

Stanford University, USA

Hasselt University, Belgium

Université Paris VII, France

Nihon University, Japan

Northeastern University, USA

University of California, San Diego, USA

\section{PRODUCTION}

contact@msp.org

Silvio Levy, Scientific Editor

See inside back cover or www.jant.org for submission instructions.

The subscription price for 2012 is US \$175/year for the electronic version, and \$275/year (+\$40 shipping outside the US) for print and electronic. Subscriptions, requests for back issues from the last three years and changes of subscribers address should be sent to Mathematical Sciences Publishers, Department of Mathematics, University of California, Berkeley, CA 94720-3840, USA.

Algebra \& Number Theory (ISSN 1937-0652) at Mathematical Sciences Publishers, Department of Mathematics, University of California, Berkeley, CA 94720-3840 is published continuously online. Periodical rate postage paid at Berkeley, CA 94704, and additional mailing offices.

ANT peer review and production are managed by EditFLOW ${ }^{\circledR}$ from Mathematical Sciences Publishers.

PUBLISHED BY

mathematical sciences publishers

http://msp.org/

A NON-PROFIT CORPORATION

Typeset in IAT $_{\mathrm{E}} \mathrm{X}$

Copyright (C2012 by Mathematical Sciences Publishers 


\section{Algebra \& Number Theory}

Volume $6 \quad$ No. $2 \quad 2012$

Arithmetic of singular Enriques surfaces

Klaus HuleK and MatThias SchÜtT

An upper bound on the Abbes-Saito filtration for finite flat group schemes and applications

YICHAO TIAN

On the smallest number of generators and the probability of generating an algebra

Rostyslav V. Kravchenko, Marcin Mazur and Bogdan V. Petrenko

Moving lemma for additive higher Chow groups

AMALENDU KRISHNA and JINHYUN PARK

CHRISTOPHER GOFF

Uniformly rigid spaces

CHRISTIAN KAPPEN

On a conjecture of Kontsevich and Soibelman

LÊ QUY THUONG 